\title{
Impact of nitrogen and carbon on defect equilibrium in $\mathrm{ZrO}_{2}$
}

\author{
H. D. Tailor, J. L. Lyons ${ }^{1}$, C.E. Dreyer ${ }^{2}$, A. Janotti ${ }^{3}$, C.G. Van de Walle* \\ Materials Department, University of California, Santa Barbara, CA 93106-5050
}

\begin{abstract}
We investigate the electronic properties of nitrogen and carbon impurities in $\mathrm{ZrO}_{2}$ using density functional theory with a hybrid functional. It is commonly accepted that $\mathrm{N}$ substitutes on the $\mathrm{O}$ site and is stable in the -1 charge state $\left(\mathrm{N}_{\mathrm{O}}^{-}\right)$. The $\mathrm{N}_{\mathrm{O}}^{-}$acceptors are then compensated by donor defects such as $\mathrm{O}$ vacancies in the +2 charge state. We test the validity of this assumption by determining the formation energies of all relevant charge states of nitrogen and oxygen vacancies as a function of Fermi level. We also examine the effects of carbon, a common unintentional impurity in $\mathrm{ZrO}_{2}$. We find that carbon impurities have relatively low formation energies and would indeed incorporate easily during growth of $\mathrm{ZrO}_{2}$, but they do not affect the equilibrium between nitrogen and oxygen vacancies. Our results show that the Kröger-Vink picture is valid for common growth conditions and the dominant defects are $\mathrm{N}_{\mathrm{O}}^{-}$and $\mathrm{V}_{\mathrm{O}}^{+2}$.
\end{abstract}

Keywords: Fuel cell materials, First-principles calculations, Yttria-stabilized zirconia polycrystal, Solid Oxide Fuel Cell (SOFC)

\footnotetext{
* Corresponding author

${ }^{1}$ Present address: Center for Functional Nanomaterials, Brookhaven National Laboratory, Upton, NY 11973

${ }^{2}$ Present address: Department of Physics and Astronomy, Rutgers, The State University of New Jersey, Piscataway, NJ 08854

${ }^{3}$ Present address: Department of Materials Science and Engineering, University of Delaware, Newark, DE 19716
}

Preprint submitted to Acta Materialia

July 1, 2016

(C) 2016. This manuscript version is made available under the Elsevier user license http://www.elsevier.com/open-access/userlicense/1.0/ 


\section{Introduction}

Zirconia $\left(\mathrm{ZrO}_{2}\right)$ is of great interest for an array of technological applications, including solid oxide fuel cells (SOFCs) [1], thermal barrier coatings in gas turbines [2], and high- $\kappa$ dielectrics in metal-oxide semiconductor field-effect transistors (MOSFETs) [3]. In SOFCs, the addition of impurities may be used for functionalization of the material and improving performance. Yttria-stabilized zirconia (YSZ) is employed in SOFCs because doping with yttrium produces oxygen vacancies to enable mobile oxygen ion conduction. Yttrium incorporates on the $\mathrm{Zr}$ site, acting as an acceptor $\left(\mathrm{Y}_{\mathrm{Zr}}^{-}\right)$. To satisfy charge neutrality, one oxygen vacancy in the +2 charge state is created for every $2 \mathrm{Y}$ ions substituted on $\mathrm{Zr}$ sites.

Oxygen vacancies are also invoked as compensation centers for impurities such as nitrogen, based on the assumption that these impurities incorporate on the oxygen site and are stable in acceptor charge states. The intentional doping of $\mathrm{N}$ in $\mathrm{ZrO}_{2}$ films has been shown to lead to increased ionic conductivity in SOFCs at high temperatures [4]. Nitrogen incorporation in $\mathrm{ZrO}_{2}$ is often described using the following mechanism,

$$
\mathrm{N}_{2}(\text { gas })+3 \mathrm{O} \rightleftharpoons 2 \mathrm{~N}_{\mathrm{O}}^{-1}+\mathrm{V}_{\mathrm{O}}^{+2}+3 / 2 \mathrm{O}_{2}(\text { gas })
$$

Here we use the notation in which the superscripts indicate the charge state of the defect or impurity [5]; in Kröger-Vink notation[6], a negative charge state would be indicated by a $/\left(\mathrm{N}_{\mathrm{O}}^{\prime}\right)$, and a positive charge state by a $\bullet\left(\mathrm{V}_{\mathrm{O}}^{\bullet \bullet}\right)$.

5 Several experimental and computational studies base their conclusions on Eq. (11) 7, 8, 9, 10], assuming that $\mathrm{N}$ is only stable in the $\mathrm{N}_{\mathrm{O}}^{-}$acceptor charge state. In the present work, we perform a comprehensive first-principles study of the nitrogen impurity in all possible configurations and all relevant charge states of $\mathrm{N}$ in $\mathrm{ZrO}_{2}$. Combining these results with results for $\mathrm{V}_{\mathrm{O}}$ allows us to assess whether the mechanism embodied in Eq. (1) is sufficient, or whether any other charge states or configurations of nitrogen need to be considered. We also study carbon impurities as they are commonly found unintentionally incorporated in 
$\mathrm{ZrO}_{2}$ thin films[11].

Previous first-principles calculations on $\mathrm{N}$ and $\mathrm{C}$ in $\mathrm{ZrO}_{2}$ were limited to neutral charge states 12, 13, 14]. In wide-band gap materials, impurities often occur in many different charge states depending on the Fermi-level position. Therefore, studying only a single charge state provides incomplete information on the behavior of the impurity. We determine the stability of the impurities by calculating their formation energies as a function of Fermi-level position in the band gap and the chemical potentials of the atomic reservoirs [5]. The previous first-principles studies on nitrogen and carbon in $\mathrm{ZrO}_{2}$ used the local density approximation (LDA) or generalized gradient approximation (GGA) within density functional theory (DFT) [12, 13, 14]. LDA and GGA severely underestimate the band gap, leading to large errors in the position of the chargestate transition levels within the band gap[5]. In the present study we use a hybrid functional, which has been proven much more accurate than traditional functionals in describing the defect formation energies and defect levels in wideband-gap materials [15].

The details of the calculation are outlined in Sec. II. Results for the configurations and the behavior of the impurities under different experimental growth conditions are discussed in Sec. III.

\section{Computational Methods}

Our calculations are based on the generalized Kohn-Sham theory with the screened hybrid functional of Heyd, Scuseria, and Ernzerhof (HSE) 16, 17] as implemented in the Vienna Ab-initio Simulation Package (VASP) [18]. The HSE mixing parameter was set to include $32 \%$ of Hartree-Fock exchange such that the calculated band gaps for tetragonal and monoclinic $\mathrm{ZrO}_{2}(5.82 \mathrm{eV}$ and 5.29 $\mathrm{eV}$, respectively) fall within the range of reported experimental values (from 5.0 to $5.8 \mathrm{eV} \mathrm{[19]).} \mathrm{We} \mathrm{have} \mathrm{found} \mathrm{that} \mathrm{this} \mathrm{mixing} \mathrm{parameter} \mathrm{well} \mathrm{describes} \mathrm{the}$ band gaps and lattice parameters of $\mathrm{ZrO}_{2}$ as well as similar oxide materials such as $\mathrm{Al}_{2} \mathrm{O}_{3}$ and $\mathrm{HfO}_{2}$ [20, 21]. The calculated lattice parameters for tetragonal 
$\mathrm{ZrO}_{2}$ are $a=3.61 \AA, c=5.18 \AA$, and for monoclinic $\mathrm{ZrO}_{2} a=5.16 \AA, b=5.21$ $\AA$, and $c=5.31 \AA$. These lattice parameters are in close agreement with the experimental values for tetragonal $\mathrm{ZrO}_{2}(a=3.61 \AA, c=5.18 \AA)$ [22] and for 45 monoclinic $\mathrm{ZrO}_{2}(a=5.15 \AA, b=5.21 \AA, c=5.32 \AA)$ [23].

The impurities are studied using the supercell approach with periodic boundary conditions [5]. We use a 108-atom supercell for tetragonal $\mathrm{ZrO}_{2}$ and a 96atom supercell for monoclinic $\mathrm{ZrO}_{2}$. The electron-ion interactions are treated using projector augmented wave potentials [24], with a cutoff of $400 \mathrm{eV}$ for the plane-wave basis set. Lattice relaxations and symmetry-broken configurations were taken into account. Spin polarization was considered for supercells containing an odd number of electrons.

Taking the nitrogen impurity on an oxygen site $\left(\mathrm{N}_{\mathrm{O}}\right)$ as an example, the formation energy in charge state $q$ is calculated as [5]:

$$
E^{f}\left(\mathrm{~N}_{\mathrm{O}}^{q}\right)=\mathrm{E}_{\mathrm{tot}}\left(\mathrm{N}_{\mathrm{O}}^{q}\right)-\mathrm{E}_{\mathrm{tot}}\left(\mathrm{ZrO}_{2}\right)-\mu_{\mathrm{N}}+\mu_{\mathrm{O}}+q \epsilon_{\mathrm{F}}+\Delta^{q},
$$

where $E_{\text {tot }}\left(\mathrm{N}_{\mathrm{O}}^{q}\right)$ is the total energy of the supercell containing $\mathrm{N}_{\mathrm{O}}$ in charge state $q$ and $E_{\text {tot }}\left(\mathrm{ZrO}_{2}\right)$ is the total energy of the bulk oxide in the same supercell. The Fermi level $\epsilon_{F}$ is referenced to the valence-band maximum (VBM). $\Delta^{q}$ is the charge-state dependent correction due to the finite size of the supercell [25, 26].

The chemical potentials $\mu_{\mathrm{N}}$ and $\mu_{\mathrm{O}}$ are referenced to the energy per atom of isolated $\mathrm{N}_{2}$ and $\mathrm{O}_{2}$ molecules, and $\mu_{\mathrm{Zr}}$ is referenced to the energy per atom of $\mathrm{Zr}$ bulk. $\mu_{\mathrm{O}}$ and $\mu_{\mathrm{Zr}}$ are treated as variables but must satisfy the stability condition of $\mathrm{ZrO}_{2}$ :

$$
\mu_{\mathrm{Zr}}+2 \mu_{\mathrm{O}}=\Delta H_{f}\left(\mathrm{ZrO}_{2}\right),
$$

where $\Delta H_{f}\left(\mathrm{ZrO}_{2}\right)$ is the formation enthalpy of $\mathrm{ZrO}_{2}$. The chemical potential $\mu_{\mathrm{N}}$ is bounded by the formation of secondary phases, in this case $\mathrm{Zr}_{3} \mathrm{~N}_{4}$ :

$$
3 \mu_{\mathrm{Zr}}+4 \mu_{\mathrm{N}} \leq \Delta H_{f}\left(\mathrm{Zr}_{3} \mathrm{~N}_{4}\right)
$$

Similarly, the upper limit of $\mu_{\mathrm{C}}$ is bounded by the formation enthalpy of $\mathrm{CO}_{2}$. The calculated formation enthalpies $\Delta H_{f}\left(\mathrm{ZrO}_{2}\right)=-10.89 \mathrm{eV}, \Delta H_{f}\left(\mathrm{Zr}_{3} \mathrm{~N}_{4}\right)=-7.74$ 
$\mathrm{eV}$, and $\Delta H_{f}\left(\mathrm{CO}_{2}\right)=-4.08 \mathrm{eV}$ are in good agreement with the experimental values[27].

The chemical potentials can be chosen to describe specific growth or processing conditions. Here we use $\mu_{\mathrm{O}}$ of an $\mathrm{O}_{2}$ gas at a given temperature and pressure to relate the results to the growth conditions of $\mathrm{ZrO}_{2}$. In the following we will present results using chemical potentials for the oxygen-rich limit $\left(\mu_{\mathrm{O}}=0\right)$ and two methods typical for the large-scale synthesis of $\mathrm{ZrO}_{2}$ for SOFC application, the co-precipitation method $\left(\mu_{\mathrm{O}}=-1.40 \mathrm{eV}\right)$ and the skull melting method $\left(\mu_{\mathrm{O}}=-2.63 \mathrm{eV}\right)[7]$. These chemical potential values were derived using the temperature and pressure conditions reported in the literature [28, 7].

In tetragonal $\mathrm{ZrO}_{2}, \mathrm{Zr}$ is eightfold coordinated by $\mathrm{O}$ atoms and $\mathrm{O}$ is fourfold coordinated by $\mathrm{Zr}$ atoms. The lengths of the $\mathrm{Zr}$-O bonds range from $2.07 \AA$ to $2.36 \AA$ A. In monoclinic $\mathrm{ZrO}_{2}, \mathrm{Zr}$ is sevenfold coordinated with $\mathrm{O}$ atoms. Three of the $\mathrm{O}$ atoms are coordinated by three $\mathrm{Zr}$ atoms and four of the $\mathrm{O}$ atoms are fourfold coordinated to $\mathrm{Zr}$ atoms. The $\mathrm{Zr}-\mathrm{O}$ bonds for threefold coordinated $\mathrm{O}$ atoms (referred to as O3 from here on) are 2.07 to $2.15 \AA$ in length, and for fourfold coordinated O atoms (O4) 2.15 to $2.24 \AA$.

\section{Results and Discussion}

\subsection{Nitrogen on a substitutional oxygen site}

We first discuss the results for the tetragonal phase. In Fig. 1 we show the formation energy as a function of Fermi level for the stable charge states of so $\mathrm{N}_{\mathrm{O}}$ in tetragonal $\mathrm{ZrO}_{2}$, at $\mu_{\mathrm{O}}=-2.63 \mathrm{eV}$ (corresponding to the skull melting method). The slope of each line corresponds to the charge state of the impurity. The Fermi-level positions where the slope changes correspond to the chargestate transition levels $\left(q / q^{\prime}\right)$. For Fermi-level positions below the transition level $\left(q / q^{\prime}\right)$ the defect is stable in the charge state $q$, and for Fermi levels above

${ }_{85}\left(q / q^{\prime}\right)$ the defect is stable in the charge state $q^{\prime}$. The charge states determine the electrical activity of the impurity. 


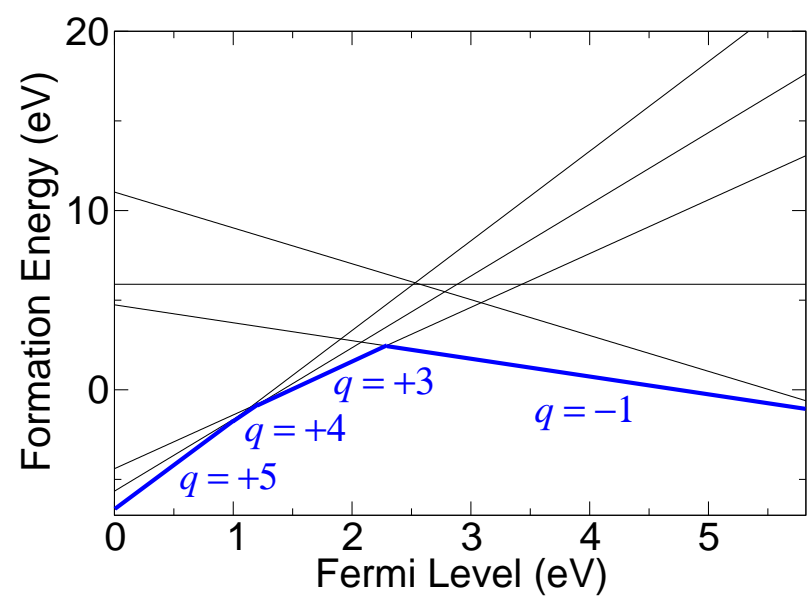

Figure 1: Formation energy as a function of Fermi level for $\mathrm{N}_{\mathrm{O}}$ in tetragonal $\mathrm{ZrO}_{2}$ in various charge states for $\mu_{\mathrm{O}}=-2.63 \mathrm{eV}$. Each line corresponds to a formation energy for a specific charge state as defined by Eq (2). The thick blue lines indicate the energetically most favorable charge state for each Fermi level in the band gap. The Fermi level is referenced to the VBM of $\mathrm{ZrO}_{2}$.

Nitrogen substituting on an $\mathrm{O}$ site is commonly assumed to be present in the neutral or -1 charge states. We indeed find that $\mathrm{N}_{\mathrm{O}}$ is stable in the -1 charge state for Fermi levels above $2.28 \mathrm{eV}$. However, for Fermi levels lower in the band gap, $\mathrm{N}_{\mathrm{O}}$ can also occur in the $+3,+4$, and +5 charge states; i.e., acting as a donor rather than an acceptor. Note that the neutral charge state is never thermodynamically stable. The presence of these positive charge states implies that the negative charge state of nitrogen might not be the only one playing a role in the reaction expressed by Eq. (1). Nitrogen could thus potentially selfcompensate, i.e., in the absence of other defects or impurities, it would appear that $\mathrm{N}_{\mathrm{O}}$ donors $\left(+3,+4\right.$ or +5 charge states) could charge-compensate the $\mathrm{N}_{\mathrm{O}}$ acceptors. This will be discussed in more detail when we compare the formation energies of $\mathrm{N}_{\mathrm{O}}$ and oxygen vacancies in Sec. 3.4

In the negative charge state, $\mathrm{N}_{\mathrm{O}}^{-}$retains the configuration of the $\mathrm{O}$ atom, 

positive charge states of $\mathrm{N}_{\mathrm{O}}$ all exhibit configurations similar to what is shown in Fig. 2(a) for $\mathrm{N}_{\mathrm{O}}^{+3}$, where the $\mathrm{N}$ atom undergoes a large off-site displacement and forms two N-O bonds similar in bond length.
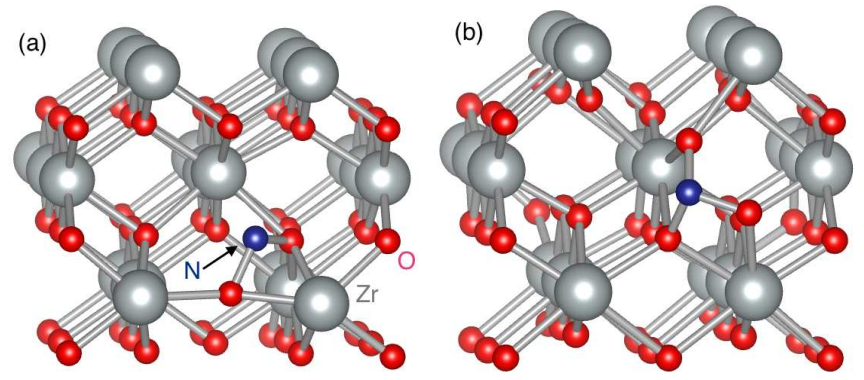

Figure 2: Atomic configuration of (a) $\mathrm{N}_{\mathrm{O}}^{+3}$ and (b) $\mathrm{N}_{\mathrm{Zr}}^{-}$in tetragonal $\mathrm{ZrO}_{2}$.

\subsection{Nitrogen in other configurations}

Figure 3 shows the formation energies of nitrogen in various configurations, for different values of the oxygen chemical potential. Some of the information that was included in Fig. 1 for $\mathrm{N}_{\mathrm{O}}$ is included in Fig. 3(a), but only the line segments corresponding to the lowest formation at each Fermi level are shown here. In addition to substituting on the oxygen site, nitrogen can substitute on the $\mathrm{Zr}$ site, or form a nitrogen interstitial.

On the cation site in tetragonal $\mathrm{ZrO}_{2}, \mathrm{~N}_{\mathrm{Zr}}$ is characterized by an offsite displacement towards three of the $\mathrm{O}$ nearest neighbors. It can occur in three different charge states: $+1,0$, and -1 . Figure 3 shows that $\mathrm{N}_{\mathrm{Zr}}$ would be stable only under very oxygen-rich conditions, which are unlikely to occur during growth. If it would incorporate, it would be mainly in the negative charge state, in which the structure is characterized by bonding of $\mathrm{N}$ to three $\mathrm{O}$ atoms with N-O bond lengths of 1.39, 1.40, and $1.45 \AA$ [see Fig. 2(b)].

The nitrogen interstitial prefers to incorporate on a site coordinated by two $\mathrm{O}$ atoms as nearest neighbors in tetragonal $\mathrm{ZrO}_{2}$. It can occur in a variety of charge states. However, as is evident from Fig. 3, its formation energy is so high 
(a)

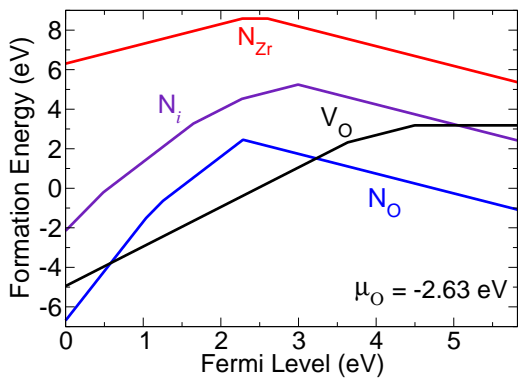

(b)

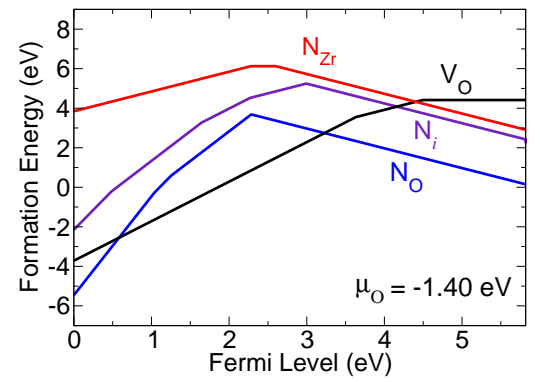

(c)

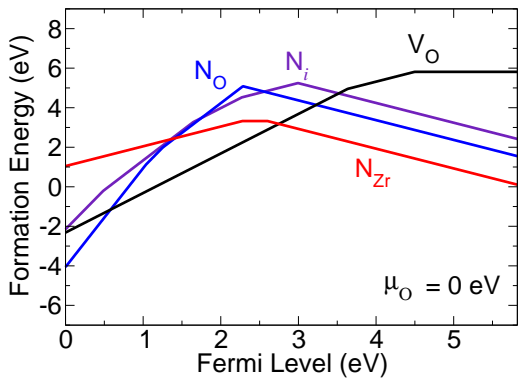

Figure 3: Formation energy as a function of Fermi level for nitrogen in various configurations in tetragonal $\mathrm{ZrO}_{2}$ for (a) $\mu_{\mathrm{O}}=-2.63 \mathrm{eV}$ (skull melting growth conditions), (b) $\mu_{\mathrm{O}}=-1.40$ $\mathrm{eV}$ (wet-chemical co-precipitation conditions), and (c) $\mu_{\mathrm{O}}=0 \mathrm{eV}$ (oxygen-rich conditions). The formation energy of oxygen vacancies is included as well.

that it is never a relevant configuration under any conditions.

\subsection{Oxygen vacancies and charge equilibrium}

We now address how nitrogen in its various configurations interacts with oxygen vacancies in $\mathrm{ZrO}_{2} . \mathrm{V}_{\mathrm{O}}$ has a $(+2 /+1)$ level $3.64 \mathrm{eV}$ and a $(+1 / 0)$ level $4.49 \mathrm{eV}$ above the VBM, implying that the defect will be in the +2 charge state for relevant Fermi levels. The position of the Fermi level is determined by overall charge neutrality. To a very good approximation, this can be obtained by inspection of the formation-energy plots, by finding the crossing point of the formation energies of the lowest-energy positively charged and negatively charged defects or impurities. This constitutes a good approximation because 

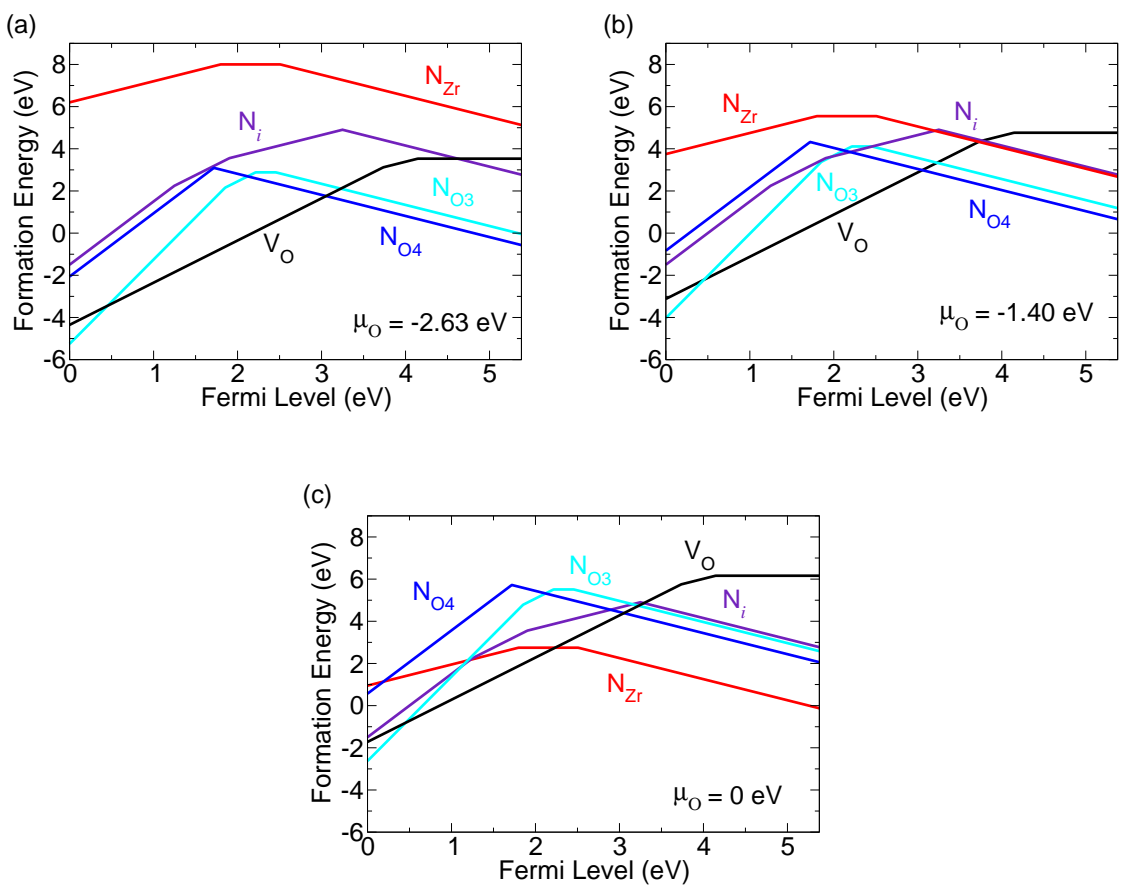

Figure 4: Formation energy as a function of Fermi level for nitrogen in various configurations in monoclinic $\mathrm{ZrO}_{2}$ for (a) $\mu_{\mathrm{O}}=-2.63 \mathrm{eV}$ (skull melting growth conditions), (b) $\mu_{\mathrm{O}}=-1.40$ $\mathrm{eV}$ (wet-chemical co-precipitation conditions), and (c) $\mu_{\mathrm{O}}=0 \mathrm{eV}$ (oxygen-rich conditions). The formation energy of oxygen vacancies is included as well.

defect concentrations decrease exponentially with increasing formation energy, and therefore the lowest-energy defect at each Fermi-level position will dominate. The formation energy of $\mathrm{V}_{\mathrm{O}}^{+2}$ is low enough that for all possible oxygen chemical potentials it constitutes the lowest-energy positive charge state that will provide charge compensation for the negatively charged nitrogen impurities. The intersection of the formation energies of $\mathrm{V}_{\mathrm{O}}$ and $\mathrm{N}_{\mathrm{O}}$ pins the Fermi level such that it is the oxygen vacancy, rather than any donor states of any of the configurations of the nitrogen impurity, that provides the charge balance in $\mathrm{ZrO}_{2}$. 


\subsection{Monoclinic $\mathrm{ZrO}_{2}$}

The results presented above were for the tetragonal phase of $\mathrm{ZrO}_{2}$. Since the structure, bonding, and band gap of the monoclinic phase are similar to those of the tetragonal phase, we expect similar behavior of the point defects and impurities, and indeed this is revealed by our calculations.

As shown in Fig. 4, for both $\mu_{\mathrm{O}}=-1.40 \mathrm{eV}$ and $\mu_{\mathrm{O}}=-2.63 \mathrm{eV}$, nitrogen on the fourfold coordinated oxygen site in monoclinic $\mathrm{ZrO}_{2}$ is the most stable nitrogen configuration. It occurs in the +3 and -1 charge states, and exhibits a $(+3 /-1)$ transition level at $1.73 \mathrm{eV}$ above the VBM. $\mathrm{N}_{\mathrm{O} 4}^{-}$is stable for Fermi levels above $1.73 \mathrm{eV}$. It behaves like the original fourfold coordinated $\mathrm{O}$ atom and has N-Zr bond lengths similar to the bond lengths in bulk $\mathrm{ZrO}_{2}$.

On the cation site in monoclinic $\mathrm{ZrO}_{2}, \mathrm{~N}_{\mathrm{Zr}}^{+}$forms N-O bonds with the three threefold coordinated oxygen atoms. The four fourfold coordinated oxygen atoms exhibit dangling bonds. The $\mathrm{N}-\mathrm{O}$ bond lengths are greater in length in the neutral and -1 charge state.

The oxygen vacancy exhibits a $(+2 /+1)$ level at $3.66 \mathrm{eV}$ and a $(+1 / 0)$ level at $4.15 \mathrm{eV}$ above the VBM, in agreement with previous hybrid functional calculations [29]. As in tetragonal $\mathrm{ZrO}_{2}$, the Fermi level is pinned in the middle of the band gap by $\mathrm{V}_{\mathrm{O}}^{+2}$ and $\mathrm{N}_{\mathrm{O}}^{-}$. The position of the Fermi level and the formation energy at the crossing point of $\mathrm{V}_{\mathrm{O}}^{+2}$ and $\mathrm{N}_{\mathrm{O}}^{-}$is similar between tetragonal and monoclinic $\mathrm{ZrO}_{2}$. Although nitrogen is also stable in positive charge states, these states are not lower in energy than $\mathrm{V}_{\mathrm{O}}^{+2}$ and hence do not affect the charge neutrality between $\mathrm{V}_{\mathrm{O}}^{+2}$ and $\mathrm{N}_{\mathrm{O}}^{-}$described in Eq. (1).

\subsection{Carbon impurities}

We now examine the potential impact of incorporation of $\mathrm{C}$ impurities, first in tetragonal $\mathrm{ZrO}_{2}$.

As shown in Fig. 5, for Fermi levels below midgap $\mathrm{C}$ on the oxygen site is stable in the +4 charge state. The atomic configuration involves $\mathrm{C}_{\mathrm{O}}$ moving offsite and forming a $\mathrm{C}-\mathrm{O}$ bond of $1.18 \AA$. In the negative charge state, $\mathrm{C}_{\mathrm{O}}$ does not move offsite and forms $\mathrm{C}-\mathrm{Zr}$ bonds similar in length to bulk $\mathrm{Zr}-\mathrm{O}$ bonds. 

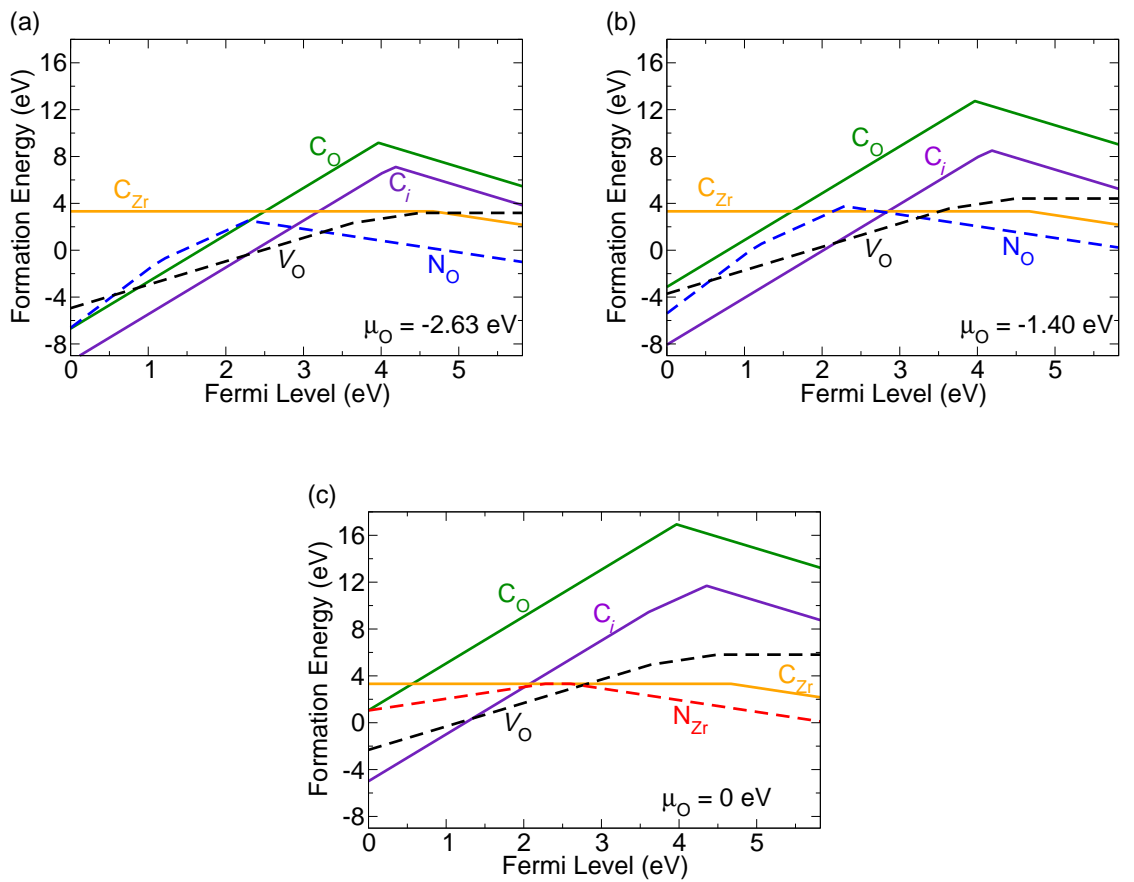

Figure 5: Formation energy as a function of Fermi level for carbon in various configurations, $V_{\mathrm{O}}$, and the lowest energy $\mathrm{N}$ defect in tetragonal $\mathrm{ZrO}_{2}$ for (a) $\mu_{\mathrm{O}}=-2.63 \mathrm{eV}$ (skull melting growth conditions), (b) $\mu_{\mathrm{O}}=-1.40 \mathrm{eV}$ (wet-chemical co-precipitation conditions), and (c) $\mu_{\mathrm{O}}=0 \mathrm{eV}$ (oxygen-rich conditions).

Carbon is isoelectronic with $\mathrm{Zr}$, and $\mathrm{C}_{\mathrm{Zr}}^{0}$ is thus expected to occur mainly in a neutral charge state. However, for Fermi levels high in the gap the -1 charge state can be stabilized, as shown in Fig. 5. Similar to $\mathrm{N}$ on the $\mathrm{Zr}$ site, $\mathrm{C}_{\mathrm{Zr}}^{0}$ enters a threefold bonding arrangement with the nearby $\mathrm{O}$ atoms, as shown in Fig. $7(a)$. The formation energy is low enough for $\mathrm{C}$ to be incorporated, possibly as an unintentional impurity, but because the dominant charge state is neutral it does not affect charge equilibration. The carbon interstitial, finally, has very low formation energies in the +4 charge state, $\mathrm{C}_{i}^{+4}$, but only for Fermi levels in the lower part of the band gap. These Fermi levels will, in general, not be accessible for growth conditions near equilibrium since $\mathrm{C}$ and $\mathrm{N}$ defects have negative formation energy there, and therefore $\mathrm{ZrO}_{2}$ will not be stable; even in 

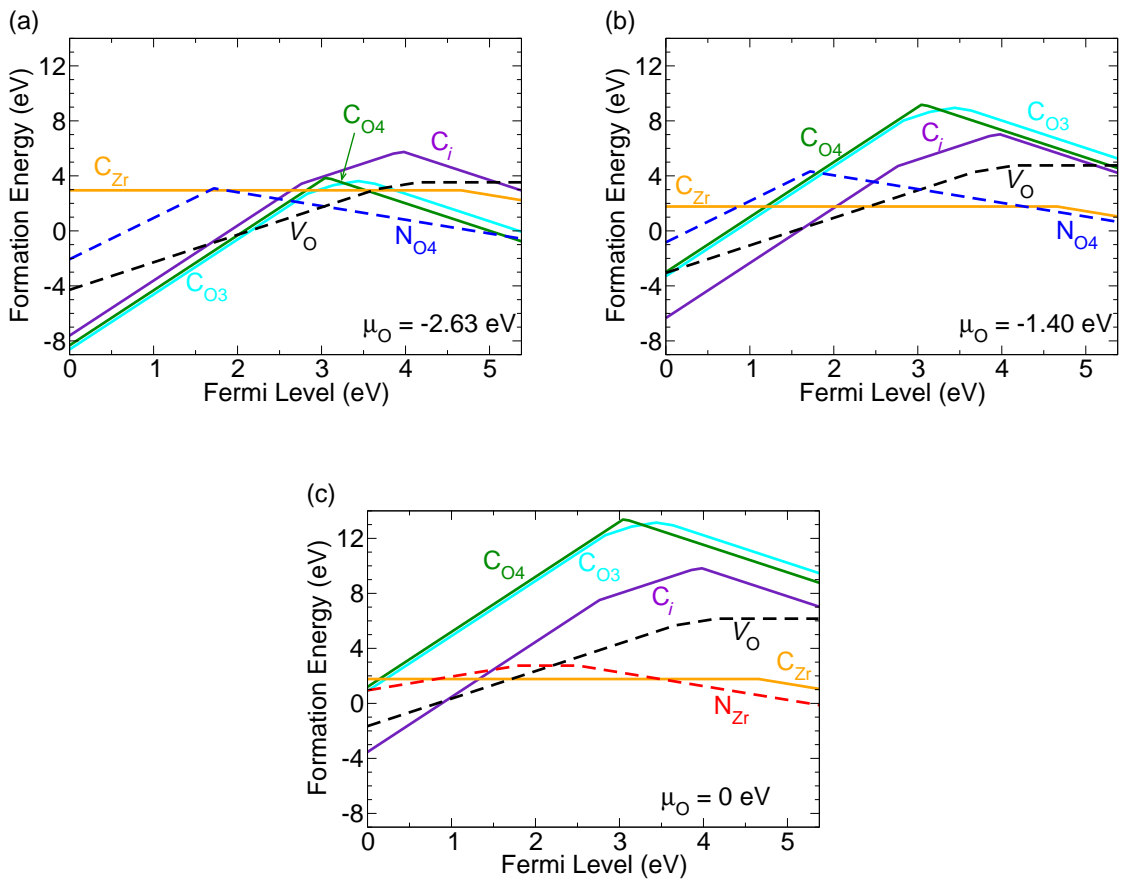

Figure 6: Formation energy as a function of Fermi level for carbon in various configurations, $V_{\mathrm{O}}$, and the lowest energy $\mathrm{N}$ defect in monoclinic $\mathrm{ZrO}_{2}$ for (a) $\mu_{\mathrm{O}}=-2.63 \mathrm{eV}$ (skull melting growth conditions), (b) $\mu_{\mathrm{O}}=-1.40 \mathrm{eV}$ (wet-chemical co-precipitation conditions), and (c) $\mu_{\mathrm{O}}=0 \mathrm{eV}$ (oxygen-rich conditions).
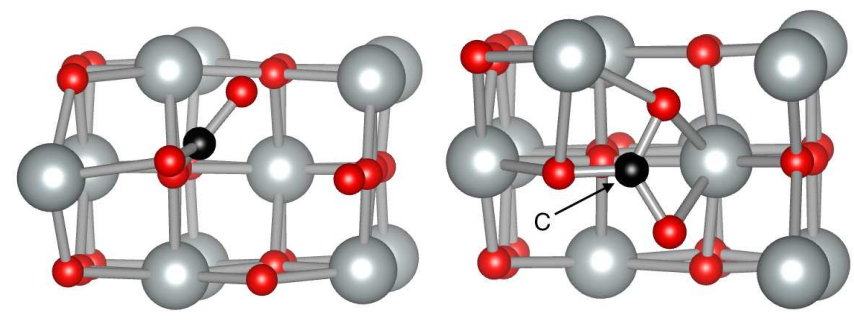

Figure 7: Atomic configurations for (a) $\mathrm{C}_{\mathrm{Zr}}$ in the neutral charge state and (b) $\mathrm{C}_{i}$ in the +4 charge state in tetragonal $\mathrm{ZrO}_{2}$.

the case of more $\mathrm{C}$ - and N-poor conditions, $V_{\mathrm{O}}$ has negative formation energy for Fermi levels near the VBM even under O-rich conditions. $\mathrm{C}_{i}^{+4}$ also enters a threefold bonding arrangement with three $\mathrm{O}$ atoms, as shown in Fig. 7 (b). 
In Fig. [5 we also plot the formation energy of the $V_{\mathrm{O}}$ and the lowest energy $\mathrm{N}$ defect for each condition (reproduced from Fig. 3). We see that for the defects and conditions considered, the Fermi level will be pinned near the mid gap by $V_{\mathrm{O}}^{+2}$ and either $\mathrm{N}_{\mathrm{O}}^{-}$or $\mathrm{N}_{\mathrm{Zr}}^{-}$. Therefore, we conclude that in tetragonal $\mathrm{ZrO}_{2}$, for all conditions considered, the presence of $\mathrm{C}$ will not affect the compensation of $V_{\mathrm{O}}^{+2}$ by $\mathrm{N}_{\mathrm{O} / \mathrm{Zr}}^{-}$assumed in Eq. (1).

The behavior of $\mathrm{C}$ in monoclinic $\mathrm{ZrO}_{2}$, shown in Fig. 6, is more sensitive to the growth conditions but similar conclusions can be reached. For $\mu_{\mathrm{O}}=-2.63$ $\mathrm{eV}$ [Fig. 6(a)], $\mathrm{C}$ on the oxygen site is stable in the +4 charge state with both $\mathrm{C}_{\mathrm{O} 3}$ and $\mathrm{C}_{\mathrm{O} 4}$ having similar formation energies. Their atomic configuration is very similar with both $\mathrm{C}_{\mathrm{O} 3}$ and $\mathrm{C}_{\mathrm{O} 4}$ moving offsite and forming two $\mathrm{C}-\mathrm{O}$ bonds of 1.29 and $1.30 \AA$. In the -1 and -2 charge state, $\mathrm{C}_{\mathrm{O} 3}$ and $\mathrm{C}_{\mathrm{O} 4}$ remain on site and form C-Zr bonds similar in length to bulk Zr-O bonds.

$\mathrm{C}_{\mathrm{Zr}}$ is again stable in the neutral charge state for most Fermi levels in the band gap, with $\mathrm{C}$ entering a threefold bonding arrangement with the nearby oxygen atoms. The $(0 /-1)$ transition level of $\mathrm{C}_{\mathrm{Zr}}$ occurs at $4.67 \mathrm{eV}$ above the VBM. Results for this charge-state transition level of $\mathrm{C}$ in monoclinic $\mathrm{ZrO}_{2}$ were previously published [30]. In Figs. 6(b) and (c), $\mathrm{C}_{\mathrm{Zr}}$ has low formation energy over the range where the Fermi level is pinned by $V_{\mathrm{O}}^{+2}$ and $\mathrm{N}_{\mathrm{Zr}}^{-}$, though it is in the neutral charge state and will not compensate the $V_{\mathrm{O}}^{+2}$.

Interstitial carbon $\mathrm{C}_{i}$ is again most stable for Fermi levels in the lower part of the band gap, with $\mathrm{C}_{i}^{+4}$ in a threefold bonding arrangement similar to that in tetragonal $\mathrm{ZrO}_{2}$.

Similar to the conclusion for the tetragonal phase, while carbon has low formation energies and may be present in high concentrations, its presence does not alter the charge neutrality between $\mathrm{V}_{\mathrm{O}}^{+2}$ and $\mathrm{N}_{\mathrm{O} / \mathrm{Zr}}^{-}$.

\section{Conclusions}

We report the electronic properties of carbon and nitrogen impurities in $\mathrm{ZrO}_{2}$ through first principles calculations. We go beyond previous first princi- 
ples studies on these defects by using a hybrid functional, which allows for more accurate predictions of formation energies and defect levels, and by comprehensively addressing all possible atomic configurations and charge states. We find that although nitrogen is present in positive charge states, the dominant defects are $\mathrm{N}_{\mathrm{O}}^{-}$and $\mathrm{V}_{\mathrm{O}}^{+2}$. This picture is consistent with the Kröger-Vink reaction in Eq. (11). Additionally, carbon impurities with their low formation energy may be present in high concentrations in $\mathrm{ZrO}_{2}$ used for SOFCs. Carbon is stable in donor charge states in $\mathrm{ZrO}_{2}$ but does not affect the charge neutrality between $\mathrm{N}_{\mathrm{O}}^{-}$and $\mathrm{V}_{\mathrm{O}}^{+2}$.

\section{Acknowledgements}

HT was supported by an NSF Graduate Research Fellowship (DGE-1144085). JL was supported by the ONR DEFINE MURI (N00014-10-1-0937). AJ was supported by the NSF MRSEC program (DMR-1121053). The work made use of the Center for Scientific Computing at the CNSI and MRL: an NSF MRSEC (DMR-1121053) and NSF CNS-0960316. This research also used resources of the National Energy Research Scientific Computing Center, which is supported by the Office of Science of the U.S. Department of Energy under Contract No. DE-AC02-05CH11231.

\section{References}

\section{References}

[1] N. Minh, Solid oxide fuel cell technology?features and applications, Solid State Ionics 174 (1-4) (2004) 271-277. doi:10.1016/j.ssi.2004.07.042

235 [2] V. Varanasi, T. Besmann, E. Payzant, B. Pint, J. Lothian, T. Anderson, High-growth rate YSZ thermal barrier coatings deposited by MOCVD demonstrate high thermal cycling lifetime, Mater. Sci. Eng., A 528 (3) (2011) 978-985. doi:10.1016/j.msea.2010.09.063. 
[3] E.-T. Kim, S.-G. Yoon, Characterization of zirconium dioxide film formed by plasma enhanced metal-organic chemical vapor deposition, Thin Solid Films 227 (1) (1993) 7-12. doi:10.1016/0040-6090(93)90179-S

[4] I. Valov, V. Rührup, R. Klein, T.-C. Rödel, a. Stork, S. Berendts, M. Dogan, H.-D. Wiemhöfer, M. Lerch, J. Janek, Ionic and electronic conductivity of nitrogen-doped YSZ single crystals, Solid State Ionics 180 (28-31) (2009) 1463-1470. doi:10.1016/j.ssi.2009.09.003.

[5] C. Freysoldt, B. Grabowski, T. Hickel, J. Neugebauer, G. Kresse, A. Janotti, C. G. Van de Walle, First-principles calculations for point defects in solids, Rev. Mod. Phys. 86 (1) (2014) 253-305. doi:10.1103/RevModPhys.86.253

[6] F. Kröger, The Chemistry of Imperfect Crystals., Wiley, 1974.

[7] D.-K. Lee, C. C. Fischer, I. Valov, J. Reinacher, A. Stork, M. Lerch, J. Janek, An EMF cell with a nitrogen solid electrolyte-on the transference of nitrogen ions in yttria-stabilized zirconia., Phys. Chem. Chem. Phys. 13 (3) (2011) 1239-42. doi:10.1039/c003991h.

[8] I. Valov, C. Korte, R. a. De Souza, M. Martin, J. Janek, Electrochemical Incorporation of Nitrogen into a Zirconia Solid Electrolyte, Electrochem. Solid-State Lett. 9 (5) (2006) F23. doi:10.1149/1.2183888.

[9] M. Lerch, Nitridation of Zirconia.

[10] M. Lerch, J. Lerch, K. Lerch, Kinetic and thermodynamic studies on the incorporation of nitrogen into zirconia, J. Mater. Sci. Lett. 15 (24) (1996) 2127-2129. doi:10.1007/BF00241147.

[11] J. Koo, Y. Kim, H. Jeon, ZrO 2 Gate Dielectric Deposited by Plasma-Enhanced Atomic Layer Deposition Method, Japanese Journal of Applied Physics 41 (Part 1, No. 5A) (2002) 3043-3046. doi:10.1143/JJAP.41.3043 
[12] G. Dutta, K. P. S. S. Hembram, G. M. Rao, U. V. Waghmare, Effects of O vacancies and $\mathrm{C}$ doping on dielectric properties of $\mathrm{ZrO} 2$ : $\mathrm{A}$ first-principles study, Appl. Phys. Lett. 89 (20) (2006) 202904. doi:10.1063/1.2388146.

[13] T. Bredow, Theoretical investigation of nitrogen substitution in cubic zirconia, Phys. Rev. B 75 (14) (2007) 144102. doi:10.1103/PhysRevB.75.144102

[14] G. Stapper, M. Bernasconi, N. Nicoloso, M. Parrinello, Ab initio study of structural and electronic properties of yttria-stabilized cubic zirconia, Phys. Rev. B 59 (2) (1999) 797-810. doi:10.1103/PhysRevB.59.797.

275 [15] P. Deák, B. Aradi, T. Frauenheim, E. Janzén, A. Gali, Accurate defect levels obtained from the HSE06 range-separated hybrid functional, Phys. Rev. B 81 (15) (2010) 153203. doi:10.1103/PhysRevB.81.153203.

[16] J. Heyd, G. E. Scuseria, M. Ernzerhof, Hybrid functionals based on a screened Coulomb potential, J. Chem. Phys. 118 (18) (2003) 8207. doi:10.1063/1.1564060.

[17] A. V. Krukau, O. A. Vydrov, A. F. Izmaylov, G. E. Scuseria, Influence of the exchange screening parameter on the performance of screened hybrid functionals., J. Chem. Phys. 125 (22) (2006) 224106. doi:10.1063/1.2404663.

[18] G. Kresse, J. Hafner, Ab initio molecular dynamics for open-shell transition metals., Phys. Rev. B 48 (17) (1993) 13115-13118.

[19] H. Jiang, R. I. Gomez-Abal, P. Rinke, M. Scheffler, Electronic band structure of zirconia and hafnia polymorphs from the GW perspective, Physical Review B 81 (8) (2010) 085119. doi:10.1103/PhysRevB.81.085119

[20] M. Choi, J. L. Lyons, A. Janotti, C. G. Van de Walle, Impact of carbon and nitrogen impurities in high- $\kappa$ dielectrics on metal-oxide-semiconductor devices, Appl. Phys. Lett. 102 (14) (2013) 142902. doi:10.1063/1.4801497. 
[21] M. Choi, J. L. Lyons, A. Janotti, C. G. Van de Walle, Impact of native defects in high-k dielectric oxides on GaN/oxide metal-oxide-semiconductor devices, Phys. Status Solidi B 250 doi:10.1002/pssb.201200628.

[22] N. Igawa, Y. Ishii, Crystal Structure of Metastable Tetragonal Zirconia up to 1473 K, J. Am. Ceram. Soc. 84 (5) (2001) 1169-1171.

[23] G. Ringanese, , J. Phys. Condens. Matter 17 (1) (2005) R357. doi:10.1116/1.4901234.

[24] P. Blöchl, Projector augmented-wave method, Phys. Rev. B 50 (24).

[25] C. Freysoldt, J. Neugebauer, C. G. Van de Walle, Fully Ab Initio Finite-Size Corrections for Charged-Defect Supercell Calculations, Phys. Rev. Lett. 102 (1) (2009) 016402. doi:10.1103/PhysRevLett.102.016402.

[26] C. Freysoldt, J. Neugebauer, C. G. Van de Walle, Electrostatic interactions between charged defects in supercells, Phys. Status Solidi B 248 (5) (2011) 1067-1076. doi:10.1002/pssb.201046289

[27] CRC Handbook of Chemistry and Physics, 84th Edition, CRC Press, Boca Raton, FL, 2003.

[28] K. Sato, G. Okamoto, M. Naito, H. Abe, NiO/YSZ nanocomposite particles synthesized via co-precipitation method for electrochemically active Ni/YSZ anode, J. Power Sources 193 (1) (2009) 185-188. doi:10.1016/j.jpowsour.2008.12.038.

[29] J. L. Lyons, A. Janotti, C. G. Van de Walle, The role of oxygen-related defects and hydrogen impurities in $\mathrm{HfO} 2$ and $\mathrm{ZrO} 2$, Microelectron. Eng. 88 (7) (2011) 1452-1456. doi:10.1016/j.mee.2011.03.099

[30] H. D. Tailor, J. L. Lyons, M. Choi, A. Janotti, C. G. Van de Walle, Carboninduced trapping levels in oxide dielectrics, J. Vac. Sci. Technol., A 33 (1) (2015) 01A120. doi:10.1116/1.4901234. 


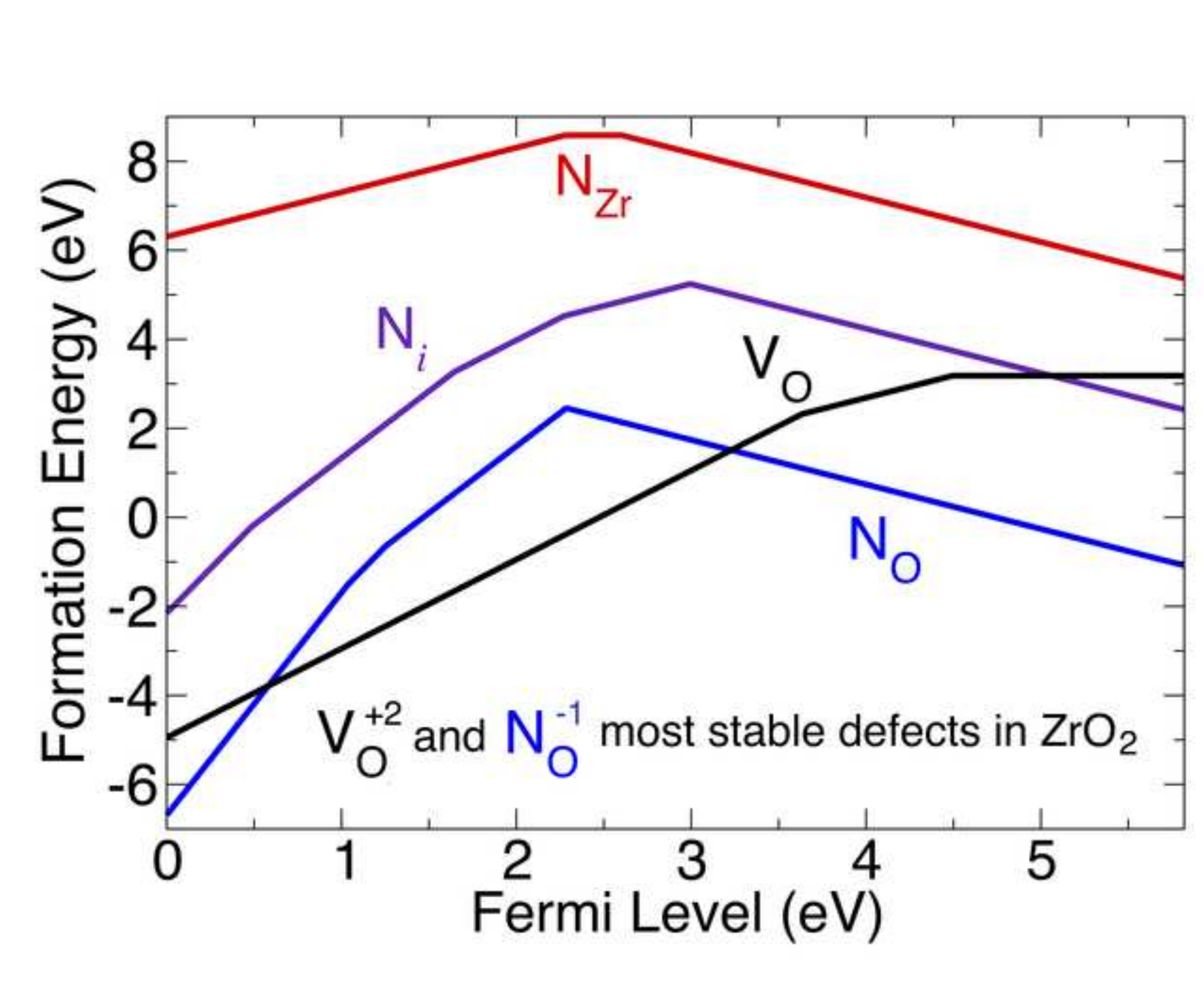

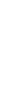

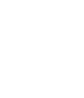

